\title{
The Param Auto Rearview Mirror a Level Surface Designs Based on Alias
}

\author{
Xiaohui Wu \\ College of Mechanical Engineering, Jilin Teacher's Institute of Engineering and Technology \\ Changchun, Jilin, China 130052 \\ xh0916@126.com
}

Keywords: Bezier; Alias; A level surface; Rearview mirror; The CAS; Detection

\begin{abstract}
This article introduced the Alias of automotive rearview mirror A level surface design content. Also explains the concept of A level surface characteristics, analyzed the meet class A surface design method of modeling steps and auto rearview mirror. Modeling scheme decision, according to three view drawing for drawing wire, physical photo, the Bezier curve, the Bezier surface requirement, choose the right solution, then, based on A level surface quality evaluation standards choose detection schemes for the class A surface.
\end{abstract}

\section{Introduction.}

The CAS surface refers to the surface with high quality, the high quality of the surface is suitable in the visual, wrinkle-free and discontinuous without reason. CAS surface in math is refers to the various points between the surface curvature radius of jumping is smaller than a certain value numerical grade and G2 continuity of the curved surface. Modelling the early stage of the design using CAS surface design department. Play a very important role for decision making and originality, CAS surface design for automotive appearance modelling style, outline of overall modelling, the proportion of car interiors and exteriors, the characteristics of automobile waist line, etc., is crucial. Automobile modeling change phase to the modelling of relevant rules and regulations, automobile design, related to engineering, the whole production manufacturing and after-sale maintenance, insurance service of checking and different proportion of automobile simulation model of sludge production, are generally by CAS surface design to assist to complete the design. We say A level Surface, it is then the CAS (Class - A - Surface) the design of the Surface, into the final used in industrial production and can meet all kinds of auto safety rules and regulations, and very precise, high quality and continuity of the curved Surface design data. A level surface is extremely important concept car design field. General requirements for G2 continuity, too small for G1 continuous fillet.

A Level Surface Conditions to be Met. A level surface for Degree and Span has strict requirements, Alias highest order for 9 order, not more than 7 order strictly, Span number is 1; For A single A surface, in UV each boundary direction to ensure consistency of curvature and ductibility, CV point distribution uniformity of change; A surface between continuous, requires at least meet tangential curvature; Surface fairing degrees, with intuitive performance should be combined with the Alias high detection, zebra crossing is checked, the Curvature color Evaluation "Curvature Evaluation" to come into the tools for Evaluation, and should not appear distorted, mutations in the inflexion of flaws. 


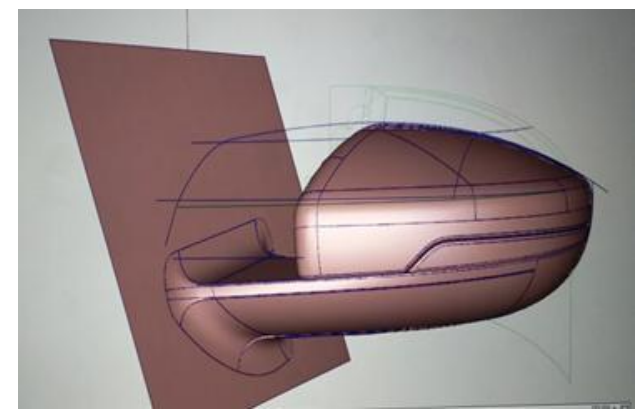

Figure 1. Rearview mirror finish figure

The Continuity of Curve. Curve, there are two main types of Nurbs curve and Bezier curve, the curve is the foundation of building surface, and can use the Skin, square, such as building surface way, Nurbs surface and the surface characteristics of the Bezier surface are major Alias, CAS surface usually use Nurbs surface modeling, class A surface usually use the Bezier surface modeling, class A surface digital model is high precision, surface quality is very good.The curve continuity category has the following four categories:

Continuous G0 - P, wire and wire joint, sharp; G1 - T continuous line and line joint is normal, but the curvature of hydrophobic size to differ, although no sharp, but fair sex is bad;G2 - C continuous, normal and curvature size are consistent, but their curvature change rate is different, the visual basic smooth transition;G3 - the rate of change of curvature is continuous and smooth continuous smoother than $\mathrm{C}$.

\section{The Car Rearview Mirror a Level Surface Structure Design}

Establish the Bezier Curve. Use less as far as possible point of CV curve is determined, need to open three depending on the size chart, according to the projection line surface of the relationship, at the same time to combine images to determine the relationship between faceted.

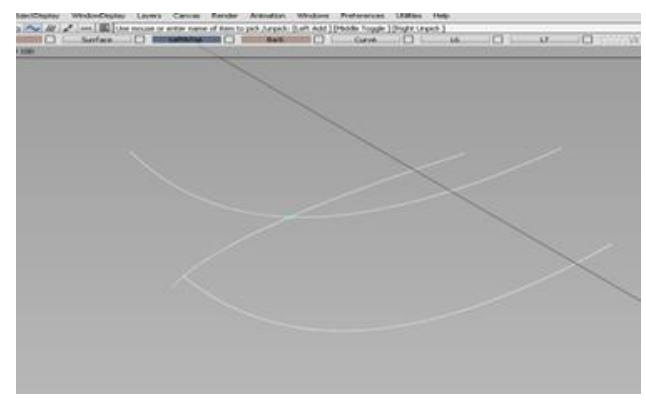

Figure 2. The Bezier curve to establish

The Bezier Surface was Established. Based on the Bezier curve, the Skin command building surface, circular arc surface need square at the top of the square into a complete, at the same time, with the help of a small keyboard vector control CV point distribution, in order to achieve the required curved continuous requirement and $\mathrm{CV}$ distribution change. Then finish other bedding face. As shown in Fig. 3, Fig. 4. 


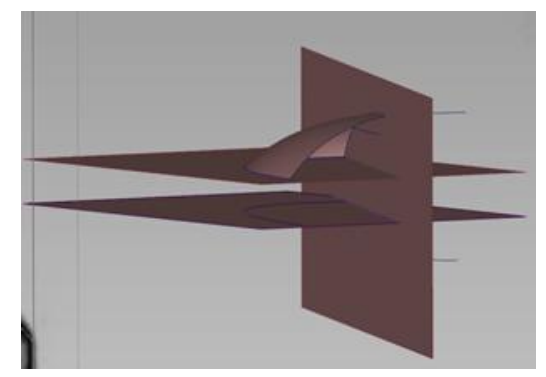

Figure 3. The Bezier surface to establish

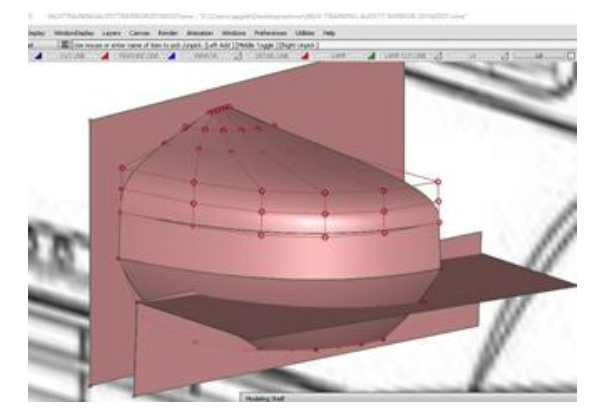

Figure 4. Adjustment of the Bezier surface $\mathrm{CV}$ point

The Bezier Surface Shear. First used the Trim Isect intersect intersection line. Began after chamfering, shear surface excess chamfering principle: after the first big then small, as shown in figure 5, is to pour the vertical this big stare blankly, then horizontal edge, here comes the trilateral manual pick Angle, in order to make the joint to $\mathrm{C}$ continuous.

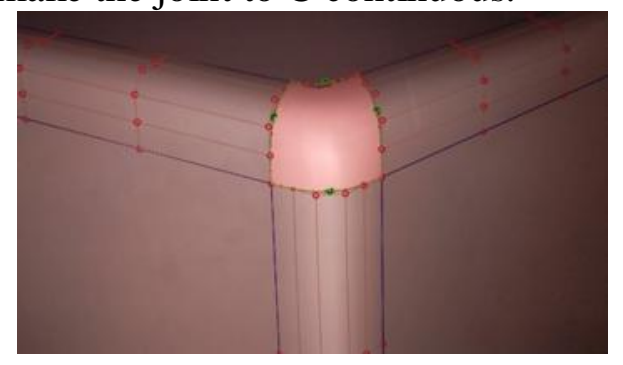

Figure 5. The trilateral manual pick Angle

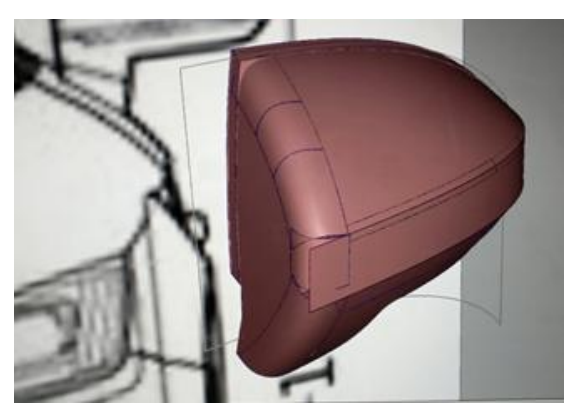

Figure 6. The shear plane, chamfering

\section{The Class A Surface Quality Evaluation Standard}

In constructing A curved surface data, on the basis of CAS surface figure, should use simple and orderly class A curved surface to express clearly and concisely highlights trends of d/A, d/A key 
characteristic line on the direction of change, all kinds of car regulations method is another issue to consider, such ability ensure the digital surface specification, reasonable, high quality, to say the product physical model of the basic is decided by A level surface digital model.

Surface Fairing. Smoothing sex is the vehicle surface quality manifested intuitively, for high visibility of the surface, especially such as roof panel, front and rear cover high reflective material part, need to keep the surface fairing, in order to gain higher visual specular effect. Surface fairing can from curvature comb, zebra crossing, evaluated for curvature coloring, etc.

Curvature comb is mathematical performance, surface curvature change of curvature comb curvature values by contour line of high and low, said the size of the tangent line is usually expressed in red line curvature comb the corresponding tangent relationship of each point on surface of the surface in the corresponding points in the direction normal direction said comb. Good surface fairing curvature comb change is regular and orderly and curvature or from large to small size or some kind of law changes. Rearview mirror curvature comb as shown in Fig. 7.

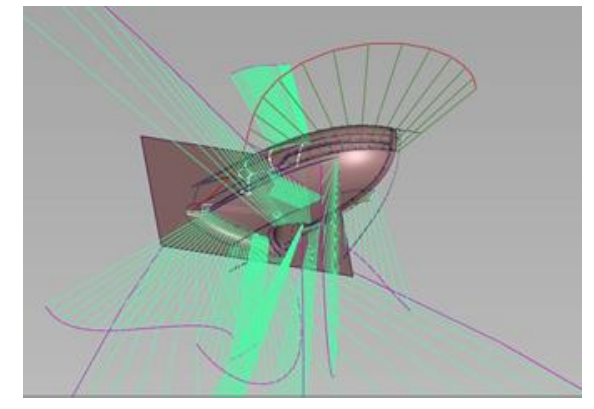

Figure 7. The curvature of the mirror hydrophobic

Zebra crossing detection, zebra crossing through the simulated surface in specular light, the color line within the launch Angle in different colors to distinguish between, and the static or dynamic specular colour separation surface figure. Zebra crossing surface requirement is a zebra crossing, regular and orderly transition smooth, without defects such as folding turn, this requires from different direction detection is achieved. A zebra crossing as shown in figure 8, shown in Fig. 9.

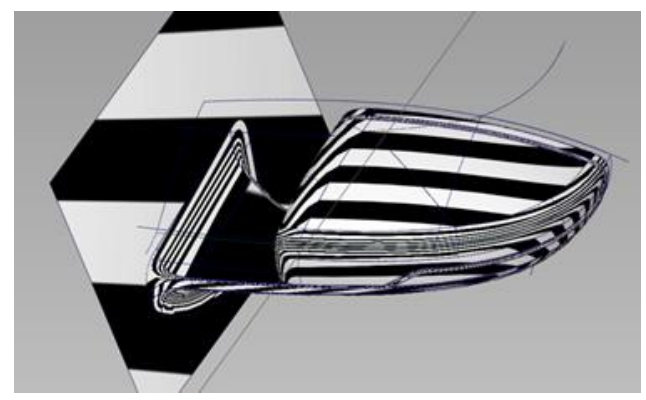

Figure 8. Overlooking the zebra crossing 


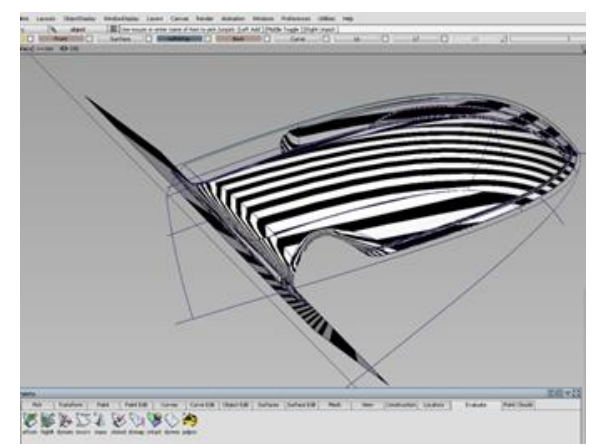

Figure 9. Look up at a zebra crossing

CV Control Points Arranged Regularly. Whether the surface smooth, shape rules depend on specifications CV point arrangement, should follow some rules:

The spacing between each CV points evenly spaced evenly spaced or change;

Each CV at the distance from the curve and curved surface distribution is reasonable and has a certain regularity, or increase or decrease the gradient rule, there is no single $\mathrm{CV}$ points above or below other $\mathrm{CV}$ point phenomenon;

The arrangement of $\mathrm{CV}$ point average curvature large area arrangement is less $\mathrm{CV}$ point, curvature small area arrangement more CV point;

The curve to the change of curvature of the surface of uniform gradient. CV point arrangement of rearview mirror as shown in Fig. 10.

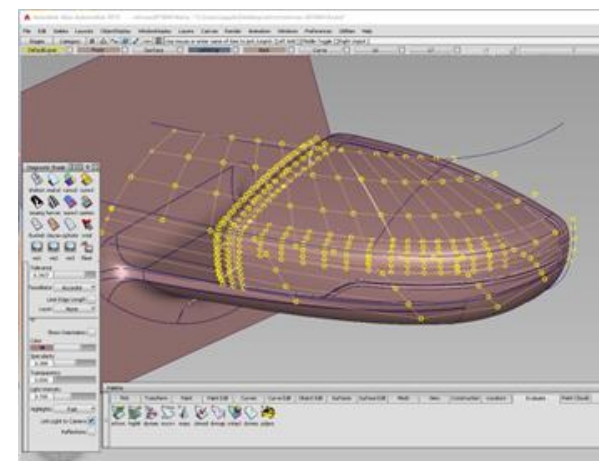

Figure 10. $\mathrm{CV}$ control point configuration rule

The ory of Surface Intersection Curve Curvature Thin Fairing. As shown in Fig. 11.

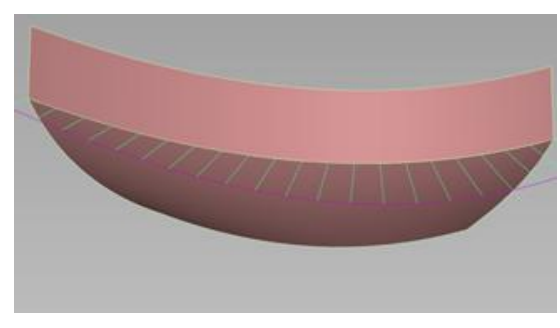

Figure 11. Intersecting line curvature hydrophobic surface theory

\section{The Knot Language}

Through the above process, first introduced the class A surface analysis and class A surface conditions to be met, involved the concept of curve, the curve there are two main types of Nurbs curve and Bezier curve, because the curve is the foundation of building surface, usually use Nurbs 
surface modeling of CAS face, A level surface usually use the Bezier surface modeling, at the same time stressed the curve continuity four categories to help to build A better line and then complete the high quality of surface, surface modeling design is ordered phase incremental work, because it is first built the thinking of the Bezier curve, and then build the Bezier surface, the Bezier surface shear, here used Trim Isect intersect intersection line get basic model. The chamfer to consider chamfering principle: first big then small, also involves the trilateral manual pick Angle, in order to make the joint to $C$ continuous. Surface design based on Alias can be A very good class A surface modelling of this demand, at the same time, the correct understanding and use of surface quality evaluation will be testing standards we can obtain the guarantee of high quality surface.

\section{References}

[1] M Li, Alias, Studio/Paint Application in the Passenger Train Modelling Design [J]. Vehicle Technology and Research, 2011, (1).

[2] B.O Yang, F.G Wang and A H, Based on The Alias of Free-form Surface Reverse Modeling Design [J]. Journal of Donghua University, 2010, (4) : 389-392.

[3] L.J Cao, X.T Zhang and L Zhao, etc. Alias and Product Modeling Design [J]. Journal of Packaging Engineering, 2007 (3).

[4] J.S Wu, Free Surface in the Automobile Body Modelling Design Rule Analysis [D]. Shanghai: Donghua University, 2007.

[5] Q Jiang, Read Digital Automobile Modeling Design Technology [J]. Journal of CAD, CAM and Manufacturing Information, 2009, (7) : 50-53.

[6] M.H Han, etc. Car Design Practical Handbook [M]. China Building Industry Press, 2009.

[7] [7]J.P Min, W Wei, Based on the Alias of Free-form Surface Automobile Modeling Design [J]. Journal of Automotive Engineers, 2011, (5).

[8] H.L Li, Chen xu. Alias Showcase Product Modeling Design Performance of Typical Case Analysis [M]. Beijing: Electronic Industry Press, 2013.

[9] B.O Yang, Product Design Using Alias Tutorial [M]. Beijing: Hydraulic Press.

[10] M.H Han, J.B Liu, Autodesk AliasStudio Car Design Practical Handbook [M]. Beijing: China Building Indåustry Press, 2009. 